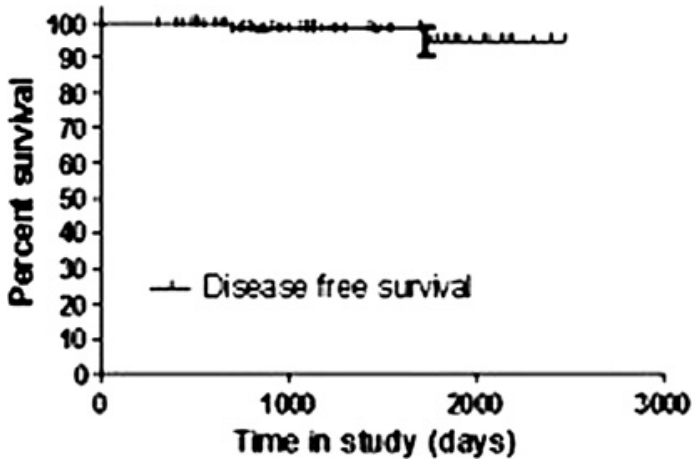

Abstract PM0-151 Figure 1 HCV free survival in patients who achieve SVR.

currently treated. Antiviral therapy for injecting drug users with $\mathrm{HCV}$ in North East London is provided by a Blood Borne Virus nursing team based in community outreach clinics. We aimed to examine HCV and drug related outcomes in patients treated by this service.

Methods A retrospective notes analysis was performed of all patients treated between September 2006 and June 2011. Data were collected on demographics, HCV treatment, health and social outcomes. Minitab 16 and Prism 5 were used to perform statistical analysis. The Wilcoxon signed rank test, unpaired $\mathrm{T}$ test, and Mann-Whitney test were used to analyse drug and alcohol and demographic outcomes.

Results 152 patients were treated. 77 were active IDU's and 75 were ex-IDU's. $80 \%$ were male. $45 \%$ were genotype $1,54 \%$ were genotype 2 or $3.81 \%$ of patients were compliant with treatment. 105 patients (69\%) achieved an end of treatment response (ETR) Sustained viral response (SVR) rate was $58 \%$. Overall survival post SVR was $100 \%$ and HCV free survival was $98 \%$. Two patients were re-infected at 14 and 51 months after treatment (See Abstract PMO-151 figure 1) five deaths occurred 21, 32, 35, 37 and 44 months after treatment finished. All deaths were in non-responders. No deaths were attributable to treatment. There was no significant difference in demographics or treatment outcomes between active and ex-IDU's. Full data on heroin injection use was available in 133 patients, crack use in 58 patients and alcohol in 72 patients. Overall heroin injection use pre- and post-treatment reduced from $51 \%$ of patients to $38 \%$ ( $<<0.0001)$, crack use reduced from $34 \%$ to $19 \%(p<0.0001)$, and alcohol use from $38 \%$ to $32 \%(p=0.0035)$.

Conclusion This is the largest study published to-date examining the impact of antiviral therapy in patients actively using illicit drugs. Of importance long term follow-up data showing low re-infection rates, a significant reduction in illicit drug use after therapy and excellent disease free and overall survival in patients who achieve SVR is presented. Our findings confirm previous, small scale studies showing that effective treatment for injectors is possible within the appropriate clinical setting.

Competing interests None declared.

\section{PM0-152 CHARACTERISING THE IMMUNE STATUS OF HBV- SPECIFIC CD4+ AND CD8 + T-CELLS PRODUCING IL-17 IN PATIENTS WITH CHRONIC HEPATITIS B (CHB) VIRUS INFECTION}

doi:10.1136/gutjnl-2012-302514b.152

${ }^{1} \mathrm{H}$ Cooksley, ${ }^{*}{ }^{1} \mathrm{~A}$ Riva, ${ }^{2} \mathrm{M}$ Simonova, ${ }^{2} \mathrm{~K}$ Katzarov, ${ }^{1} \mathrm{~S}$ Chokshi. ${ }^{1}$ Institute of Hepatology, Foundation for Liver Research, London, UK; ${ }^{2}$ Clinic of Gastroenterology \& Hepatology, Military Medical Academy, Sofia, Bulgaria

Introduction Virus specific CD4+ and CD8+T-cells are essential in the control of HBV infection and their functions are tightly regu- lated by immune homeostatic control mechanisms, such as the programmed death (PD1) pathway, Tim3 and CD244. These pathways maintain the equilibrium between efficient control of viral replication and unnecessary inflammatory/immunopathological damage. Recent studies have described a unique subset of T-cells which produce IL-17. Preliminary studies suggest that IL-17producing $\mathrm{T}$-cells maybe involved in inflammation and liver damage but largely the role of HBV-specific IL-17 producing CD4+(Th17) and $\mathrm{CD} 8+(\mathrm{Tc} 17)$ T-cells during chronic HBV infection remains elusive. Moreover, the impact of immunoregulatory signatures on these T-cells are unknown. The aim of this study was to characterise the role and immune status of virus-specific CD4+ Th17 and CD8+ Tc17 cells in CHB patients.

Methods Peripheral blood mononuclear cells were collected from ten treatment naïve $\mathrm{HBeAg}+\mathrm{CHB}$ patients and ten healthy controls. PBMC's were stimulated with recombinant $\mathrm{HBcAg} / \mathrm{HBsAg}$ and $\mathrm{PMA}$ /Ionomycin. The frequency of total and virus-specific CD4 and CD8 T-cell producing IL-17/IFNg and the expression of T-cell immunoregulatory molecules PD1, Tim3 and CD244 was analysed by flow cytometry.

Results Total number of CD8+ T-cells producing IL-17 was not different between chronic HBV patients and healthy controls. However HBV-specific Tc17 cells were significantly higher in CHBpatients compared to controls $(p=0.007)$. Total Th17 were also higher in CHB-patients compared to controls $(p=0.03)$ however the difference was more pronounced in $\mathrm{HBV}$-specific CD4+ Th-17 $(p=0.003)$. Upon analysis of the immune-homeostatsis signatures we found a higher expression of PD1 and CD244 on HBV-specific $\mathrm{CD} 4+$ and CD8 + T-cells producing IFNg $(p<0.001$ and $p=0.026$ respectively) in $\mathrm{CHB}$ patients. No expression of Tim3 was found on these cells. However, HBV-specific Th17 cells in the CHB patients did not express PD1 or CD244 but had levels of Tim3 significantly lower than healthy controls $(p=0.027)$.

Conclusion This study reveals the involvement of virus-specific Th17 and Tc17 in the pathogenesis of chronic HBV infection. Interestingly, we observe differential patterns of immunoregulatory signatures operational within the populations of virus-specific $T$ cells producing IFNg and IL-17 which may influence their role in HBV disease.

Competing interests None declared.

\section{PM0-153 HEPATITIS A VIRUS VACCINATION IN PERSONS WITH HEPATITIS C VIRUS INFECTION: CONSEOUENCES OF IMPLEMENTATION IN LOW INCIDENCE AREAS}

doi:10.1136/gutjnl-2012-302514b.153

${ }^{1,2} \mathrm{~A}$ A Rowe, ${ }^{* 1,2} \mathrm{R}$ Parker, ${ }^{1,2} \mathrm{M} \mathrm{J}$ Armstrong, ${ }^{1,2} \mathrm{D} \mathrm{D}$ Houlihan, ${ }^{1,2} \mathrm{D}$ Mutimer. ${ }^{1}$ Centre for Liver Research and NIHR Biomedical Research Unit, University of Birmingham, Birmingham, UK; ${ }^{2}$ Liver and Hepatobiliary Unit, Queen Elizabeth Hospital, Birmingham, UK

Introduction Hepatitis A virus (HAV) superinfection in persons with hepatitis $C$ virus (HCV) infection has been associated with a high mortality rate. Consequently HAV vaccination is recommended by many authorities for this patient group. The incidence of HAV is low and has been reducing in many areas, including Western Europe and the USA. The aim of this study was therefore to determine the cost and clinical consequences of routine vaccination in persons with HCV infection.

Methods To determine the mortality risk of HAV superinfection a meta-analysis including studies reporting mortality in HCV infected persons was done using RevMan 5.1 (Cochrane Collaboration). Data were extracted independently by two investigators and recorded on a standardised spreadsheet. The pooled mortality estimate was used 